\title{
Impact of Penetration of Photovoltaic on Rotor Angle Stability of Power System
}

\author{
Jitendra Thapa, Dr. Sanjeev Maharjan
}

\begin{abstract}
The small signal and transient behaviour of large multi-machine system is minimal when the small- scale PV farm power is connected to it. But the oscillation on dynamics or states of the multi-machine system begins to increase as the large-scale PV farm power is connected. In this work investigations are carried out on small signal stability and transient of large multi-machine system with PV connected to it. Eigenvalue analysis is carried out for both cases with and without the PVs and are compared. Transient stability is analysed by time domain simulation by executing three phase short circuit fault at critical location. When PV are integrated without the replacement of conventional generators, small signal stability and transient stability both improves. Eigenvalue analysis shows that the damping of critical modes increases. And the magnitude of the peak of rotor angle of generator 2 decreases from 17.984o to 13.0690 due to addition of PV which shows improvement in transient stability. In contrast, the stability of system in terms of small signal and large signal both degrades as the addition of $\mathrm{PV}$ is done by the replacement of the conventional generators. The trend of critical mode 3 towards the RHP (right half plane) of the complex plane indicates that the small signal stability is degrading. For the same case, the peak of the speed of generator 1 increases form 1.015 p.u to 1.0311 p.u from no $P V$ to various level of $P V$ which indicates the degradation of transient stable. The reduction in inertia due to replacement of synchronous generators reduces the damping of the critical modes which is also verified by the transient analysis.
\end{abstract}

Index Terms - Critical clearing time, damping, eigenvalues, photovoltaic, small signal stability, and transient stability..

\section{INTRODUCTION}

Solar energy is considered one of the most propitious renewable resources and an alternative to fossils fuel. Being the universal source of energy and its high potential to contribute to the portion of world's energy, grid-connected solar farms are increasing day by day.

Along with the promising nature, the sophistication with the interconnection of PV into the grid may present several technical and major impacts on stability of the system because of its different nature to that of synchronous generators. Renewable resources are replacing conventional energy resources but side by side, the problems in integrating renewable energy in the existing grid has been the major concern. High PV penetration levels can significantly affect the steady state as well as the transient stability of the system due to their distinct characteristics that differ from the conventional generation resources.

Jitendra Thapa, Dept. of Mechanical Engineering, Institute of Engineering, Pulchowk Campus, Tribhuvan University, Pulchowk Lalitpur, Nepal, +977-9847226543

Dr. Sanjeev Maharjan, Dept. of Mechanical Engineering, Institute of Engineering, Pulchowk Campus, Tribhuvan University, Pulchowk Lalitpur, Nepal, +977-9843240144
Solar generation only contributes the active power to the system and has zero or very much reduced reactive power generation. Also, replacing conventional synchronous generators with the PV decreases the grid inertia which results in the poor damping of the system [1]. It is significant aspect of conventional synchronous generators that helps in the inertial response during frequency control [2].

Modal analysis was performed in order to analyze the small signal stability of the system in [3]. The conclusion drawn by the authors was the detrimental impact on eigenvalues as a result of reduction of inertia due to high solar-PV penetration. Damping of inter-area mode was found to be decreased due to impact of inverter-based generation on the small signal stability [4]. The authors in [5] has revealed the impact of both utility-scale and roof-top PV on power system transient stability of a large test system. The conclusion shows that solar PV could have both negative and positive impact on the transient stability of the system depending upon the fault location and the duration of fault.

In this paper, the impact of solar-PV generation on rotor angle stability is investigated using the standard IEEE 14 bus modified test system. Small-signal stability was analyzed using the Eigen value analysis method as it could analyze frequency, amplitude, damping of various oscillatory modes following a small-disturbance in the network [6]. Time domain simulation are carried out in order to evaluate the transient stability as it could capture effect on system factors such as speed and rotor angle of generators, bus voltages etc.

\section{METHODOLOGY}

\section{A. Small Signal Stability Analysis by Eigen Value Method} Small-signal stability is the ability of the power system to maintain synchronism when subjected to small disturbances [7]. In this context, a disturbance is considered to be small if the equations that describe the resulting response of the system may be linearized for the purpose of analysis. Lack of synchronizing torque result in steady increase in generator rotor angle and insufficient damping torque results in oscillation of increasing amplitude. These two above mentioned are the reasons behind instability of the system. Most practical power system suffers small signal instability due to inadequate damping of system oscillation. Smallsignal analysis utilizing linear techniques provides valuable information about the inherent dynamic characteristics of the power system and assists in its design [7].

Considering the perturbation to be small from the equilibrium point, the non-linear differential equation that represents the system dynamics can be linearized as follow,

$$
\dot{X}=A X+B U
$$


where $\mathrm{X}$ is the state vector of the system, $\mathrm{U}$ is the input vector, and $\mathrm{A}$ is the state matrix. The state vector of the system includes the states of the system such as the speed and relative rotor angles of the machines. The stability of the linearized system is determined by the eigenvalues $(\lambda)$ of the state matrix A while the participation of each system state in a specific eigenvalue is determined by the right eigenvector $(\phi)$ and the left eigenvector $(\psi)$. The $i_{\text {th }}$ eigenvalue of the system matrix $\mathrm{A}$ and its corresponding eigenvectors are defined as:

$$
\begin{aligned}
& A \Phi_{i}=\lambda_{i} \Phi_{i} \\
& \Psi_{i} A=\lambda_{i} \Psi_{i}
\end{aligned}
$$

For a complex eigenvalue that corresponds to an oscillatory mode of the system, the mode frequency in $\mathrm{Hz}$ (f) and the damping ratio $(\zeta)$ are expressed as,

$$
\begin{gathered}
\lambda_{i}=\sigma_{i} \pm j \omega_{i} \\
f_{i}=\frac{\omega_{i}}{2 \Pi} \\
\zeta i=-\frac{\sigma_{i}}{\sqrt{\sigma_{i}^{2}+\omega_{i}^{2}}}
\end{gathered}
$$

The damping ratio, which is related to the real part of the eigenvalues, in fact determines the rate at which the amplitude of the oscillations decreases. Hence, a positive real part corresponds to oscillations with increased amplitudes while a negative real part corresponds to a damped oscillation. As the complex mode moves towards the right half plane (RHP), the damping of the system worsens.

For the purpose of study following procedures are followed:

1) Critical modes of oscillation are determined by conducting eigen value analysis in the base case (no PV generation).

2) Best location for integrating single PV is determine based on the steady state analysis.

3) Scenario 1: Integrate PV in the location determined through the steady state analysis and other buses without replacing conventional generators and perform eigen value analysis with various level of penetration.

4) Scenario 2: Integrate the PV by replacing the existing generators and synchronous compensators which are used for reactive compensation and perform the eigenvalue for various level of penetration of PV.

5) Compare the results of the eigenvalue analysis under different PV penetration levels to investigate the impact of PV penetration on small signal stability.

6) Verify the result obtained from the small signal stability analysis using transient stability analysis.

\section{B. Transient Stability}

Transient stability is the ability of the power system to maintain synchronism when subjected to a severe transient disturbance such as a fault on transmission facilities, loss of generation, or loss of a large load. The consequences to such disturbances involve large deviation of generator rotor angles, power flows, bus voltages, and other system variables. The system remains in synchronism if the resulting angular separation between the machines in the system remains within the limit. Loss of synchronism because of transient instability, if it occurs, will usually be evident within 2 to 3 seconds of the initial disturbance [7].

For transient stability analysis, three phase short circuit fault is given at critical location that will excite the critical modes obtained in small signal stability analysis.

\section{TEST SYSTEM AND SOLAR PV GENERATOR}

\section{A. Modified IEEE 14 Bus System}

In this study, the dynamic IEEE 14 bus standard test system is used. Single line diagram of IEEE 14 Bus Test system is shown in Fig.1.

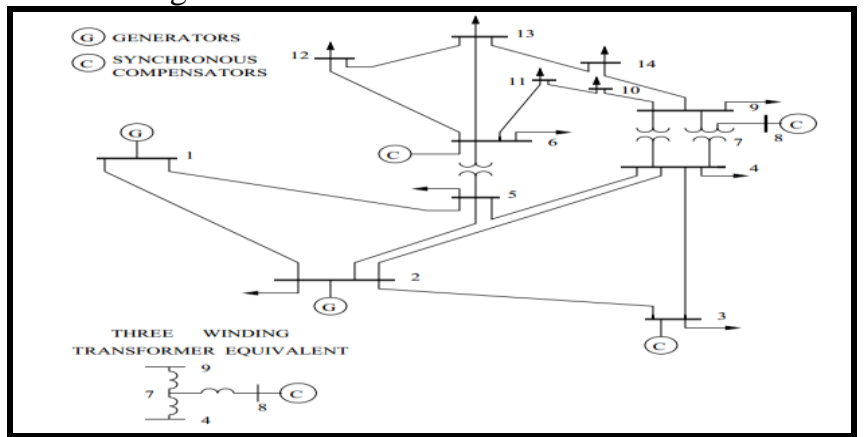

Fig. 1: Single Line Diagram of IEEE 14 Bus System [8]

Table I: Summary of the test system

\begin{tabular}{|c|c|c|}
\hline \multirow{2}{*}{ Total Load } & MW & 259 \\
\cline { 2 - 3 } & MVAr & 73.5 \\
\hline \multirow{2}{*}{ Total Generation } & MW & 271.83 \\
\cline { 2 - 3 } & MVAr & 81.27 \\
\hline \multirow{2}{*}{ Total Losses } & MW & 12.83 \\
\cline { 2 - 3 } & MVAr & 26.02 \\
\hline Total Number of Generators & \multicolumn{2}{|c|}{5} \\
\hline Total Number of Buses & \multicolumn{2}{|c|}{19} \\
\hline Total Number of Lines & \multicolumn{2}{|c|}{16} \\
\hline
\end{tabular}

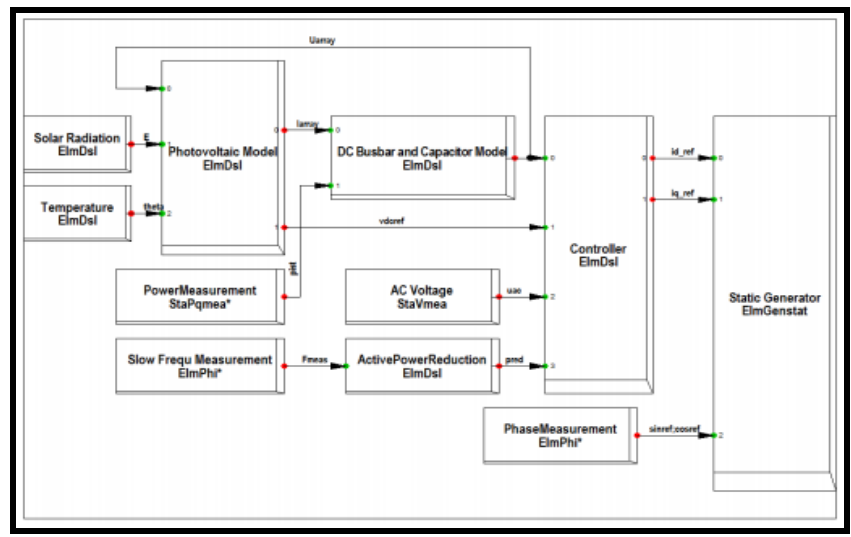

Fig. 2: The dynamic model of solar PV System [9]

Some modification are made in the IEEE 14 bus test system for study such as two winding transformers, controllers like AVR (Automatic Voltage Regulator) and governors are added to the generators. Table-I represent the features of IEEE 14 bus modified test system. 
Small signal stability as well as transient analysis is carried out on an IEEE 14 bus modified test system. Solar PV plant has been integrated into the system.

\section{B. Solar PV System}

The solar PV generation is represented by an aggregated solar-PV model using the dynamic solar-PV available in the DigSILENT PowerFactory with necessary modification. The required numbers of $\mathrm{PV}$ panels and inverters are connected in parallel to generate the required active power level. A schematic diagram of the DigSILENT PowerFactory dynamic solar-PV model is shown in Fig. 2.

The dynamic simulation model of the solar-PV system is developed with main four blocks; the photovoltaic block, the DC busbar, PQ controller and the static generator model. In this study, the dynamic solar PV is operated in active power model for generating required active power by adjusting the number of PV panels, inverter and their rating.

\section{RESULTS AND DISCUSSION}

\section{A. Steady State Analysis}

The modified IEEE 14 bus system model without solar PV integration has been considered as the base case. Bus 3, 4 and 14 are considered for the integration of PV as the largest loads are connected to these buses. The level of active power through the PV is gradually increased and injected at each bus 3, 4 and 14 separately. At each penetration level, load flow calculation is carried out and various parameters such as bus voltages and total loss of the system are noted. The penetration level is based on the percentage of the total MW demand of the system. The level of penetration is calculated as:

PV Penetration $(\%)=\mathrm{PV}$ generation $/$ Total load

Table II: Summary of the penetration level

\begin{tabular}{|c|c|c|c|c|}
\hline PV Penetration (MW) & 25.9 & 51.8 & 77.7 & 103.6 \\
\hline $\begin{array}{c}\text { PV Penetration level \% } \\
\text { load based }\end{array}$ & $10 \%$ & $20 \%$ & $30 \%$ & $40 \%$ \\
\hline
\end{tabular}

Based on the steady state analysis by penetrating the PV in bus 3, 4 and 14, the optimum penetration level and favorable location for the integration is determined. The voltage profile is improved and the system loss is minimum when the PV is integrated in bus 4 compared to the other case. MW loss starts to increase right after $20 \% \mathrm{PV}$ when integrating PV in bus 14 which is shown in Fig. 6. The MVAr loss starts to increase after the $40 \%$ of PV is injected in bus 4 which indicates the optimum level of penetration in bus 4 . So, the best location of the integration of PV is bus 4 and the maximum penetration is $40 \%$ of the total demand which is shown in Table-III. The voltage profile and system losses when the penetration level of PV is increased at each of the three buses are shown in Fig. 3 to Fig. 7.

Table III: Summary of Steady State Analysis

\begin{tabular}{|c|c|c|}
\hline Case based on & Best Location & $\%$ of Penetration \\
\hline Bus Voltage & Bus 4 & $40 \%$ \\
\hline System Loss & Bus 4 & $40 \%$ \\
\hline
\end{tabular}

Voltage Profile of different buses with different level of penetration at Bus 3

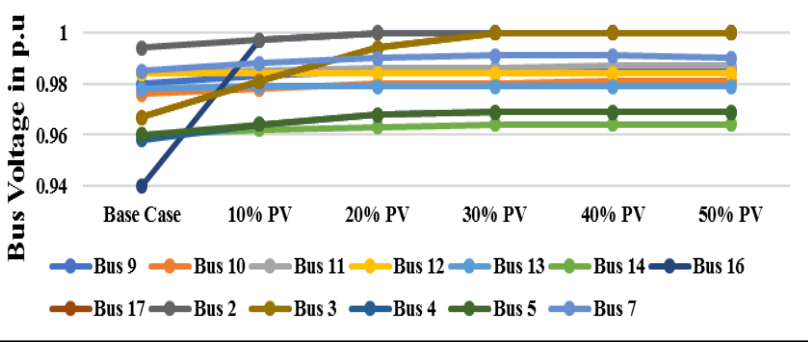

Fig. 3: Voltage Profile of differen buses with different level of penetration at bus 3

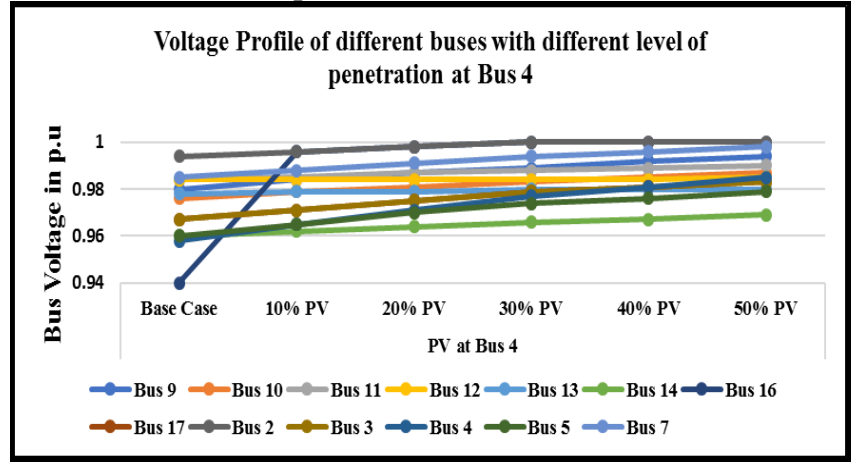

Fig. 4: Voltage Profile of differen buses with different level of penetration at bus 4

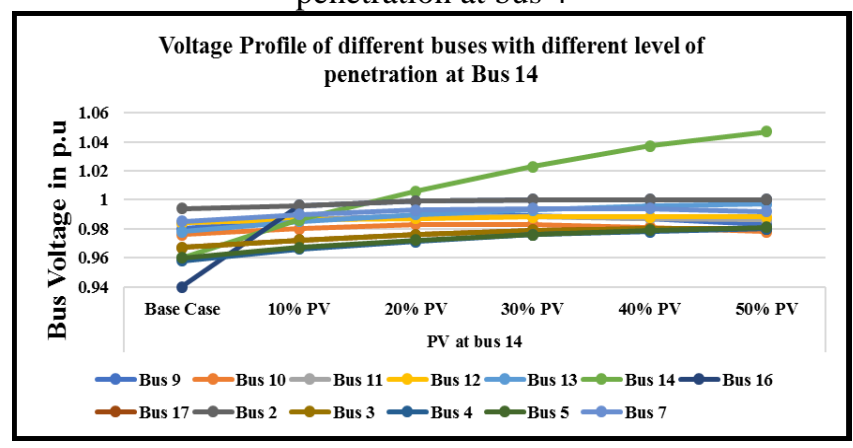

Fig. 5: Voltage Profile of differen buses with different level of penetration at bus 14

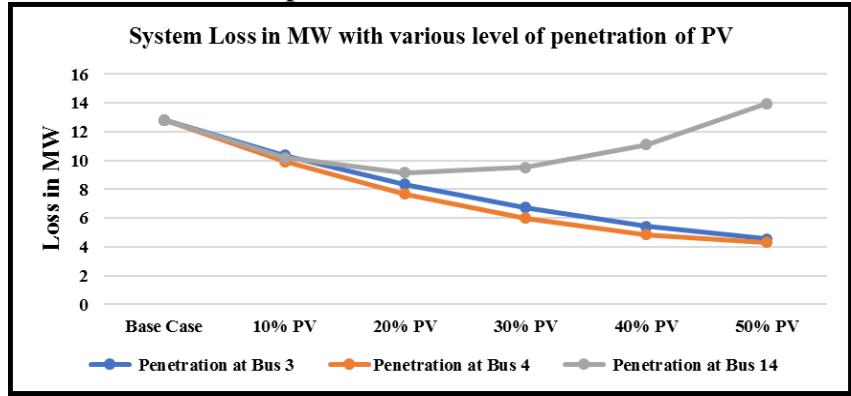

Fig. 6: MW loss profile with different level of penetration

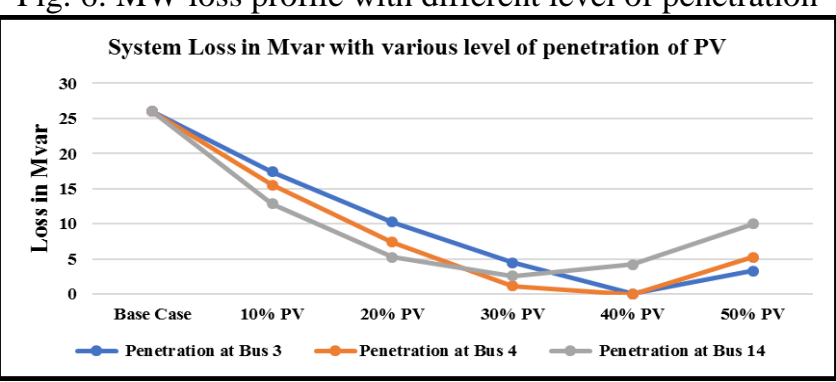

Fig. 7: MVAr loss profile with different level of penetration 


\section{B. Small Signal Stability Analysis}

1) Scenario 1: Integration of PV at bus 4 without the replacement of Generators.

Small signal stability analysis is carried out to evaluate the stability of the system under various PV penetration levels. To determine the critical modes of the system, an eigenvalue analysis is conducted for the modes with damping of less than $15 \%$. This analysis is performed utilizing the Small Signal Analysis Toolbox which is part of the DigSILENT software package. The analysis is conducted for various PV penetration levels defined in Table II. The critical modes with their damping of the base case $(0 \% \mathrm{PV})$ that are also present in all PV penetration cases are presented in Table IV and V. It can be observed that, when solar-PV penetration increases without replacing the conventional generator of the system the real-part of the eigenvalues moved away from the imaginary axis indicating an improvement in small-signal stability. The improvement in small signal stability can be also observed by the increment in damping of three critical modes 1, 2 and 3 . The damping ratio of the critical mode 1, 2 and 3 are improved by $12.38 \%, 12.48 \%$ and $3.8 \%$ respectively from no PV to $40 \%$ of PV increment. Furthermore, the damping ratio has also increased which makes the system more stable as the oscillation after the disturbance dies out faster.

2) Scenario 2: Integration of $P V$ at different buses by replacing the conventional generators

In this small signal stability study, conventionalgenerators and synchronous compensators which were used of reactive power compensation are replaced by the PV. As, the number of conventional generators are replaced, the number of modes of the system decreases. So, mode 3 which is detrimentally impacted and present in all the cases is used for the study of small signal stability.

As seen from the table VI, mode 3 is critically impacted as the penetration level increases from no PV to various level of PV by replacing conventional generators. The eigen value analysis shows that replacement of conventional generator with an aggregate solar PV model decreases the small signal stability of the system which is substantiated by the transient stability analysis as well. The eigen value of critical mode 3 shifts from $-0.184 \pm \mathrm{j} 1.1513$ to $-0.1426 \pm \mathrm{j} 1.696$ which shows the trend of mode 3 towards the RHP. The frequency of oscillation of critical mode 3 increases from 0.2408 to 0.27 $\mathrm{Hz}$ and the damping ratio decreases from 0.1208 to 0.0837 i.e. decreases by $30.71 \%$. The real part of mode decreases which indicates that the mode is shifted towards the RHP of the complex plane which indicates that the addition of PV results in decrease of small signal stability of power system. The increment in frequency of oscillation and reduction of damping of the mode is a clear indication of system being less stable for small perturbation. Fig. 8 shows clearly that the addition of PV with the replacement of generators results in poor damping.

Table IV: Critical modes with various level of penetration

\begin{tabular}{|c|c|c|c|}
\hline Case & Mode 1 & Mode 2 & Mode 3 \\
\hline $0 \%$ PV & $-1.605 \pm \mathrm{j} 14.94$ & $-1.127 \pm \mathrm{j} 13.62$ & $-0.184 \pm \mathrm{j} 1.5129$ \\
\hline $10 \% \mathrm{PV}$ & $-1.1634 \pm \mathrm{j} 14.47$ & $-1.134 \pm \mathrm{j} 13.35$ & $-0.1857 \pm \mathrm{j} 1.5132$ \\
\hline $20 \% \mathrm{PV}$ & $-1.1651 \pm \mathrm{j} 14.03$ & $-1.144 \pm \mathrm{j} 13.09$ & $-0.1875 \pm \mathrm{j} 1.5135$ \\
\hline $30 \% \mathrm{PV}$ & $-1.1654 \pm \mathrm{j} 13.63$ & $-1.159 \pm \mathrm{j} 12.84$ & $-0.1894 \pm \mathrm{j} 1.5136$ \\
\hline $40 \% \mathrm{PV}$ & $-1.1655 \pm \mathrm{j} 13.33$ & $-1.177 \pm \mathrm{j} 12.63$ & $-0.1914 \pm \mathrm{j} 1.5135$ \\
\hline
\end{tabular}

Table V: Damping of Critical Modes with various level of penetration

\begin{tabular}{|c|c|c|c|}
\hline Case & Mode 1 & Mode 2 & Mode 3 \\
\hline $0 \%$ PV & 0.0775 & 0.0825 & 0.1208 \\
\hline $10 \%$ PV & 0.0801 & 0.0846 & 0.1218 \\
\hline $20 \%$ PV & 0.0828 & 0.0871 & 0.1229 \\
\hline $30 \%$ PV & 0.0852 & 0.0899 & 0.1241 \\
\hline $40 \%$ PV & 0.0871 & 0.0928 & 0.1254 \\
\hline
\end{tabular}

Table VI: Critical Mode 3 including its frequency and damping with various level of penetration

\begin{tabular}{|c|c|c|c|}
\hline Case & Mode 3 & $\begin{array}{c}\text { Frequenc } \\
\mathbf{y}\end{array}$ & Damping \\
\hline Base case & $-0.1840 \pm \mathrm{j} 1.513$ & 0.2408 & 0.1208 \\
\hline Gen 2 replaced & $-0.1686 \pm \mathrm{j} 1.627$ & 0.2590 & 0.1031 \\
\hline Gen 2 and 3 replaced & $-0.1567 \pm \mathrm{j} 1.661$ & 0.2643 & 0.0939 \\
\hline Gen 2, 3 and 6 replaced & $-0.1495 \pm \mathrm{j} 1.678$ & 0.2672 & 0.0887 \\
\hline Gen 2, 3, 6 and 8 replaced & $-0.1426 \pm \mathrm{j} 1.696$ & 0.2700 & 0.0837 \\
\hline
\end{tabular}

\section{Damping of critical mode 3 with various level of penetration of $\mathbf{P V}$}

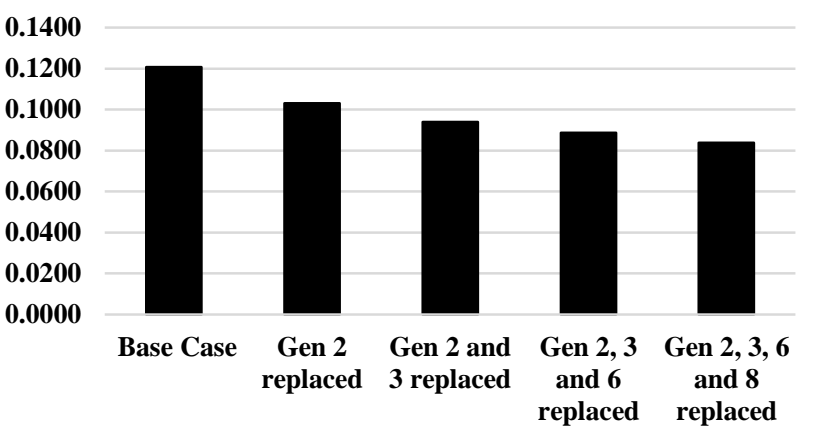

Fig 8: Damping of Critical Mode 3 with various level of penetration by replacing conventional generators

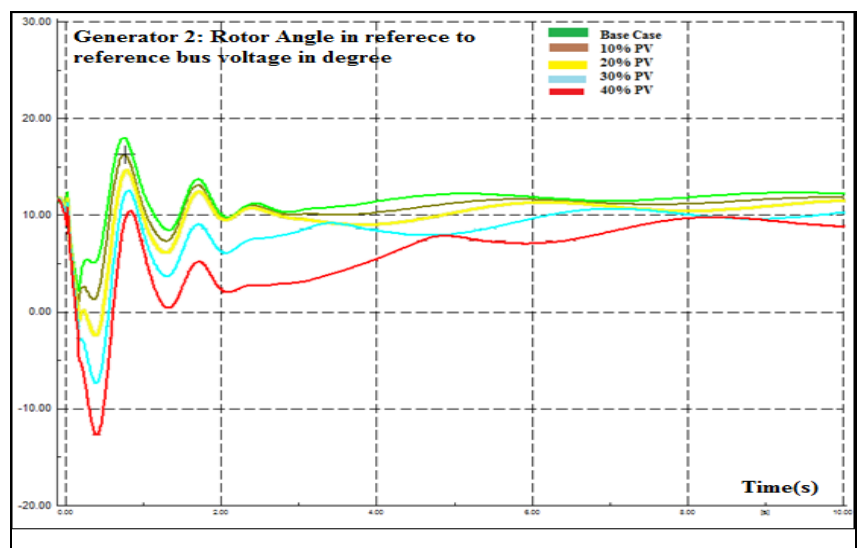

Fig. 9: Rotor Angle of generator 2 with various level of penetration of $\mathrm{PV}$

\section{Transient Stability Analysis}

In order to corroborate the results obtained from small signal stability analysis, same scenario as in the small signal stability analysis are created for transient stability analysis.

\section{1) Scenario 1: Integration of $P V$ without the} replacement of Generators.

Transient stability denotes how fast a power system can reach to its stable condition after the large disturbance. The paper 
discusses transient stability of power system with the addition of PV generation in terms of transient voltage of buses, speed and rotor angle of generators. For the purpose of analysis, three phase short circuit fault is executed on the line 2-4 at the midpoint of the transmission line.

During transient, the magnitude of the peak of oscillation of generator 2 rotor angle after fault is decreased from $17.984^{\circ}$ to $13.069^{\circ}$ from no PV to $40 \%$ of PV increment in the system without the replacement of conventional generators which can be seen in Fig. 9. Thus, the result shows that the transient stability improves as the level of PV increases when the existing conventional generators still contributing in the power production.

\section{Scenario 2: Integration of $P V$ at different buses by replacing the conventional generators}

Critical mode 3 was detrimentally impacted during the analysis of small signal stability corresponding to the case of integration of PV by replacing conventional generators. So, the detrimentally impacted mode i.e. mode 3 is scrutinized in time domain.

In order to relate the critical mode 3 with the transient analysis, the disturbance that excites it with respect to the PV penetration level should be simulated. For the transient case that would excite a detrimentally impacted critical mode, the dominant machine with the highest participation factor in that mode needs to be identified. The participation factors of various machines participating in the studied mode 3 , i.e. $-0.184 \pm \mathrm{j} 1.5291$, is shown in Fig. 10. As seen from Fig. 10, among the machine generators 1 and generator 2 have the

participation in this mode. Generator 2 is replaced by the PV generators so only the participation of the generator 1 is considered for the purpose of analysis.

Based on the participation factor, generator 1 speed is the factor affecting the mode 3. So, the disturbance that impact the speed of the generator 1 will eventually affect the critical mode 3 . So, the decrease in damping of critical mode 3 in the small signal stability analysis can be verified if the magnitude of oscillation and the number of oscillations of generator 1 speed increases when the disturbance is given to the system. The disturbance near to the generator 1 will most impact the speed of the generator 1 .

The critical location for giving three phase fault is determined based on the calculation of critical clearing time. Critical location determined based on critical clearing time is shown in Table VII. Three phase short circuit is given to the three transmission lines separately which is connected to the generator 1 and the critical clearing time is calculated for each case. Higher the critical clearing time lesser critical is the location of fault. So, three phase short circuit fault is given to the line 1-2(1) very close to the generator i.e. $0.05 \mathrm{~km}$ away from the generator 1 . The generator 2 was replaced by an aggregated model of PV generator and at $\mathrm{t}=0 \mathrm{sec}$, a three-phase short circuit fault is executed in line 1-2(1). The fault was cleared after $150 \mathrm{~ms}$. The speed of the generator 1 which is related to mode 3 and the active power produced by the generator 1 are plotted on Fig. 11 and Fig. 12 respectively. The simulations were repeated by increasing the amount of PV penetration by replacing generators at buses-17, 19, and
18 sequentially with PV generation. As the machines connected to these buses are synchronous compensators, reactive power is fed by the addition of shunt capacitor of proper rating. Also, the load of same MW as generated by PV on that same bus is increased to make a proper comparison on the active power of generator 1 . The simulation results showing the active power and speed of generator 1 for each case are plotted on Fig. 11 and Fig. 12. The transient stability shows that speed of generator 1 after penetration of PV results in increment in magnitude and oscillation which signifies the poor damping of the system which is a result of reduction in overall inertia. The peak of the generator 1 speed increases from 1.015 p.u to 1.0311 p.u with no PV to various level of PV respectively. Magnitude of the peak of speed of generator 1 and oscillations are increased which demonstrate the system being less transiently stable. The change in active power contributed by the generator 1 can be observed in Fig. 12 .

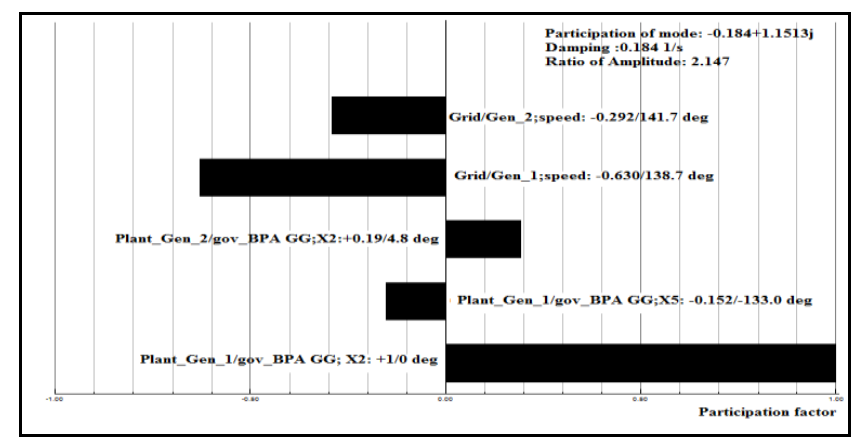

Fig. 10: Participation of Generator 1 and 2 on critical mode 3 Table VII. Critical Clearing time with location of fault

\begin{tabular}{|c|c|c|}
\hline Location of fault & Critical Clearing Time & Generator 1 speed (p.u) \\
\hline Line 1-5 & $280 \mathrm{~ms}$ & 1.03 \\
\hline Line 1-2 (1) & $221 \mathrm{~ms}$ & 1.0341 \\
\hline Line 1-2 (2) & $221 \mathrm{~ms}$ & 1.0341 \\
\hline
\end{tabular}

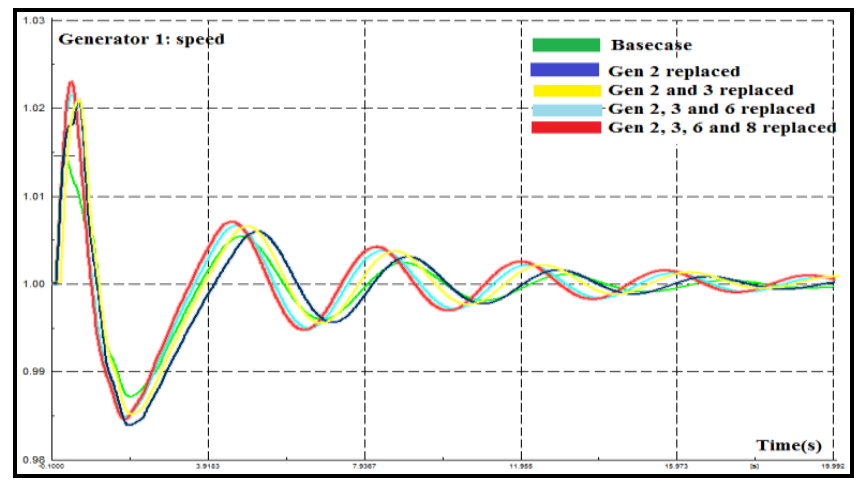

Fig. 11: Speed of generator 1 with various level of penetration

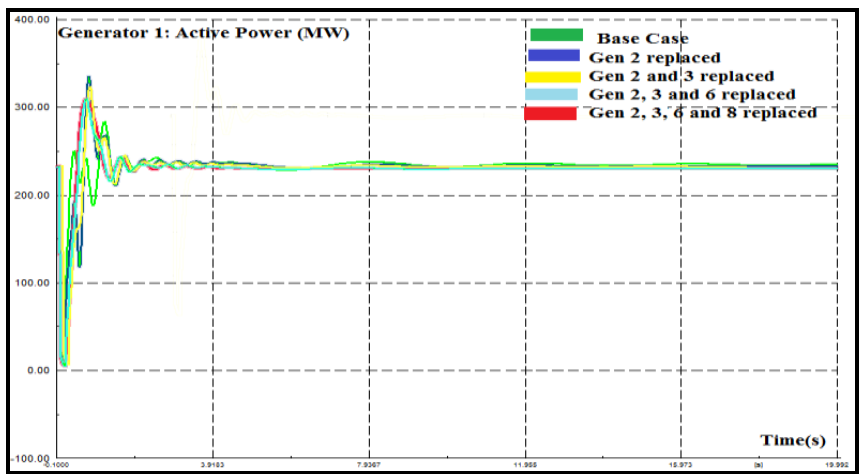

Fig. 12: Active Power of Generator 1 with various level of penetration 


\section{CONCLUSION}

When PV generation is increased without the replacement of conventional generators, both small signal stability and the transient stability of the system is improved. Eigenvalue analysis show that the damping of critical modes 1,2 and 3 increases by $12.38 \%, 12.48 \%$ and $3.8 \%$ respectively. The magnitude of the peak of rotor angle of generator 2 decreases from $17.984^{\circ}$ to $13.069^{\circ}$ due to addition of PV which shows improvement in transient stability.

While the replacement of conventional generators with the addition of PV makes the system less stable regarding both small and large signal perturbation. The trend of critical mode 3 towards the RHP of the complex plane indicates that the small signal stability is degrading because of the replacement of conventional generators by the PV generators. For the same case, the peak of the speed of generator 1 increases form 1.015 p.u to 1.0311 p.u which indicates the system is less transiently stable. Also, frequency of oscillation is increased.

The stability of the system depends upon various factors such as level of penetration of PV, fault clearing time, location of fault, and so on. So, it is imperative to determine their effect on the stability of the system. When the concentrated loads are replaced by the distributed load in the system, the eigen value changes. So, it is recommended to further investigate the effect of load distribution on the system stability.

\section{REFERENCES}

[1] J. von Appen, M. Braun, T. Stetz, K. Diwold and D. Geibel, "Time in the Sun: The Challenge of High PV Penetration in the German Electric Grid," in IEEE Power and Energy Magazine, vol. 11, no. 2, pp. 55-64, March-April 2013.

[2] U. Andreas, T.S. Borsche and G. Andersson, "Impact of Low Rotational Inertia on Power Sysem Stability and Operation," IFAC World Congress 2014, Capetown, South Africa, 2014.

[3] S. Eftekharnejad, V. Vittal, G. T. Heydt, B. Keel, and J. Loehr, "Small signal stability assessment of power systems with increased penetration of photovoltaic generation: A case study," IEEE Trans. Sust. Energy, vol. 4, pp. 960-967, 2013.

[4] Quintero, V. Vittal, G. T. Heydt and H. Zhang, "The Impact of Increased Penetration of Converter Control-Based Generators on Power System Modes of Oscillation," in IEEE Transactions on Power Systems, vol. 29, no. 5, pp. 2248-2256, Sept. 2014.

[5] E. Munkhchuluun, L. Meegahapola and A. Vahidnia, "Impact on rotor angle stability with high solar-PV generation in power networks," 2017 IEEE PES Innovative Smart Grid Technologies Conference Europe (ISGT-Europe), Torino, 2017,pp.1-6.doi: 10.1109/ISGTEurope.2017.8260229

[6] J. F. Hauer, "Application of Prony analysis to the determination of modal content and equivalent models for measured power system response," in IEEE Transactions on Power Systems, vol. 6, no. 3, pp. 1062-1068, Aug. 1991.

[7] P. Kundur et al., "Definition and classification of power system stability IEEE/CIGRE joint task force on stability terms and definitions," IEEE Trans. Power Systems, vol. 19, no. 3, pp. 1387-1401, Aug. 2004.

[8] N. Mithulananthan, C. A. Ca nizares, and J. Reeve. "Indices to Detect Hopf Bifurcation in Power Systems". In Proc. Of NAPS-2000, pp. 15-18-15-23, October 2000.

[9] M. Farhan, "Improving the Photovoltaic Model in PoweFactory," Electric Power System, 2012

[10] S. Eftekharnejad, V. Vittal, G. T. Heydt, B. Keel, and J. Loehr, "Impact of increased penetration of photovoltaic generation on power systems,'IEEE Trans. Power Syst., vol. 28, no. 2, pp. 893-901, May 2013. 